\title{
Jochen Vollmann (2008) Patientenselbstbestimmung und Selbstbestimmungsfähigkeit Beiträge zur Klinischen Ethik
}

\author{
Kohlhammer Verlag, Stuttgart, 268 Seiten, 38,00€ \\ ISBN 978-3-17-019842-5
}

\section{Claudia Wiesemann}

Online publiziert: 12. Januar 2010

(C) Die Autor(en) 2010. Dieser Artikel ist auf Springerlink.com mit Open Access verfügbar.

Seit nahezu 20 Jahren forscht und publiziert der Bochumer Psychiater und Medizinethiker Jochen Vollmann zu den ethischen Fragen der Patientenselbstbestimmung. Sein Ausgangspunkt ist dabei stets die Situation am Krankenbett, in der Patienten und Kliniker gemeinsam folgenreiche Entscheidungen fällen müssen. Das Selbstbestimmungsrecht des Patienten - obgleich in der Theorie als fundamentales Prinzip der Bioethik unumstritten - wirft in der Praxis stets noch zahlreiche Fragen auf: Wie soll Selbstbestimmungsfähigkeit als praktisches Vermögen konzeptualisiert werden? Wann ist Selbstbestimmungsfähigkeit bei Minderjährigen oder psychisch Kranken gegeben? Welchen Stellenwert hat die Selbstbestimmung des Patienten bei Entscheidungen um Leben und Tod? Auf all diese Fragen wurden in von Jochen Vollmann geleiteten Forschungsprojekten und Arbeitsgruppen empirisch fundierte Antworten gesucht, die nun in diesem Band zu einer Gesamtschau der Selbstbestimmung des Patienten aus klinisch-praktischer Perspektive zusammengestellt worden sind. Als Originalbeiträge sind sie in der Zeit von 1996 bis 2006 in deutschsprachigen Zeitschriften und Sammelbänden erschienen und damit hinreichend aktuell. Ein Aufsatz aus Health Care Analysis wurde ins Deutsche übersetzt. Als Koautoren einzelner Beiträge fungieren Armin Bauer, Torsten Marcus Breden, Heidi Danker-Hopfe, Heinz-Dieter Hartung, Hanfried Helmchen, Eva Herrmann, Klaus-Peter Kühl, Jan Schildmann und Amely Tilmann. In der Zusammenschau in Form dieses Buches bieten die Aufsätze einen fundierten und umfassenden Einblick in die praktischen Probleme der Anwendung des Konzepts der Selbstbestimmung im klinischen Alltag.

Das Eingangskapitel versammelt drei Beiträge zu den historischen und konzeptionellen Fragen des Begriffs der Selbstbestimmung. Vollmann begeht dabei nicht den Fehler der amerikanischen Bioethik, die Geschichte des Konzepts der Selbstbestimmung erst nach 1945 beginnen zu lassen. Aus seiner Feder (zusammen mit dem kürzlich verstorbenen Medizinhistoriker Rolf Winau) stammen die 1996 in Lancet und British Medical Journal erschienenen Aufsätze über die frühe rechtliche Regelung des Informed consent in der Forschung in Preußen, die das historische (Selbst-)Bild der Bioethik nachgerade revolutionierten.

C. Wiesemann $(\bowtie)$

Göttingen, Deutschland

E-Mail: cwiesem@gwdg.de 
Das zweite Kapitel diskutiert verschiedene Konzeptionen der Selbstbestimmungsfähigkeit (relationales bzw. kognitionsbasiertes Modell). Besondere Aufmerksamkeit erhält dabei die Frage der Feststellung der Selbstbestimmungsfähigkeit von Kindern. Im dritten Kapitel sind eine Reihe von empirischen quantitativen Studien zur Aufklärung und Einwilligung in der Psychiatrie versammelt. Dabei geht es vor allen Dingen um schizophrene, depressive und demente Patienten und die sich bei diesen Patienten stellenden Probleme einer Determination der Grenzen von Selbstbestimmungsfähigkeit. Aus den USA stammende Testverfahren - wie der Hopkins Competence Assessment Test (HCAT) und verschiedene MacArthur Competence Assessment Tests (MacCAT) - werden diskutiert und für den deutschen Kontext operationalisierbar gemacht. In einer Reihe von klinischen quantitativen Studien zeigen Vollmann et al. die Möglichkeiten und Grenzen dieser Testverfahren bei psychiatrischen Krankheitsbildern auf. Im letzten Kapitel werden Fragen der Selbstbestimmungsfähigkeit am Lebensende diskutiert. Vorgestellt werden insbesondere eine qualitative Erhebung der Einstellung von AIDS-Patienten in den USA zu Fragen der Sterbehilfe und des assistierten Suizids sowie eine Meta-Analyse von Studien zur Einstellung von Psychiatern zu diesen Fragestellungen.

Der Band demonstriert auf eindrückliche Weise die Relevanz und Reichweite dezidiert klinischer Fragestellungen im Feld der Medizinethik. Die vorwiegend empirisch fundierten Einzelbeiträge zeigen auf, wie sich Ethik und Empirie wechselseitig bereichern können. Für den/die Praktiker/in ist die kritische Diskussion von Instrumenten zur Feststellung der Selbstbestimmungsfähigkeit in der Praxis von großem Interesse, die im Anhang erstmalig in deutscher Version abgedruckt sind. Es ist dem Autor hoch anzurechnen, dass er die Übersetzung und Validierung dieser praktischen Instrumente in Angriff genommen hat. Wenngleich der Band - den vorwiegenden Interessen Jochen Vollmanns entsprechend - eine gewisse Psychiatrie-Lastigkeit aufweist und zudem keine Originalbeiträge enthält, kann er doch nicht weniger als eine gute Übersicht über die grundlegenden klinisch-praktischen Fragen der Selbstbestimmungsfähigkeit des Patienten einerseits und eine Anleitung zur Feststellung derselben im Klinikalltag andererseits bieten.

Open Access Dieser Artikel unterliegt den Bedingungen der Creative Commons Attribution Noncommercial License. Dadurch sind die nichtkommerzielle Nutzung, Verteilung und Reproduktion erlaubt, sofern der/die Originalautor/en und die Quelle angegeben sind. 